\title{
Development Method of Immersive Virtual Maintenance Training System
}

\author{
ZhaoKun YANG, XingXin LI, Peng WANG \\ Department of Equipment Command and Management \\ Shijiazhuang Mechanical Engineering College \\ Shijiazhuang, China \\ adanielkun@yeah.net, ${ }^{b} I x x \_1226 @$ sina.com, ${ }^{\mathrm{C}} 649797948 @ q q . c o m$
}

Keywords: virtual maintenance training; MeiTeng platform; immersive

\begin{abstract}
In view of the shortcomings of traditional equipment maintenance training, a development method of immersion virtual maintenance training system based on MeiTeng Platform of Virtual Maintenance Training is proposed. This system uses 3ds Max to build equipment model. Unity 3D is used to complete animation, interactive design with external devices. At last, the development is finished in MeiTeng Platform of Virtual Maintenance Training. We have realized this system with HTC Vive. The immersive virtual maintenance training system can meet the needs of virtual maintenance training and has good interactivity.
\end{abstract}

\section{INTRODUCTION}

As an important part of equipment life management, the importance and necessity of equipment maintenance training have been more and more obvious. With the development of information technology, equipment has been more complex, more expensive and updated faster. Compared to traditional maintenance training, virtual maintenance training has obvious advantages such as being safer, having larger capacity, better immersion and being free from time or space. The using of virtual maintenance training system can improve the ability of independent maintenance training and promote the development of maintenance support capacity.

Compared with the desktop virtual reality system, immersion virtual reality system can isolate the users' senses from the outside world, which makes it easier for users to form lasting attention and less susceptible to external interference. Applying it to the field of maintenance training can improve the quality of training, enhance the training interest and save training expenditure.

This paper presents a development method of immersive virtual maintenance training system based on MeiTeng Platform of Virtual Maintenance Training. It realizes immersive interaction with HTC Vive. The system is accomplished through the modeling of equipment based on 3ds Max, the realization of interaction based on Unity 3D and the establishment of virtual maintenance training process based on MeiTeng platform.

\section{BuILding EquipMENT Models BASEd on 3DS MAX}

Compared with other modeling software, 3ds Max creates smaller model, runs faster and has outstanding versatility. As a result, we select 3ds Max as a production tools of three-dimensional model. The establishment process and precautions of three-dimensional model are as follows.

In order to ensure the participants get real feelings and improve the training effect, the model should 
be constructed realistically as far as possible in the immersion virtual maintenance training system. In the process of building the model, the size, space position, assembly relationship and color of the virtual components should be consistent with the actual components. In order to reduce the burden of memory operation and improve the efficiency of simulation, it is necessary to simplify the modeling standards of the components which are not associated with virtual maintenance training subjects. Such as we do not construct the screw thread when we build a screw model. In addition, we are supposed to complete the modeling of maintenance tools, including hands, screwdriver, wrench, pliers and so on.

\section{A. Building the Basic Model}

The basic appearance is built through standard primitives and extended primitives. The assembly between components and the size of components should be considered as well. The assembly of models should be built according to the levels of equipment. Such as Cylinder block- Cylinder block assembly Diesel engine - Power system.

\section{B. Editing the Model Marerial}

Open the material editor and edit the material of the specified object. The specific material should be consistent with the actual situation, including the physical properties, special effects, deformer parameters, the basic parameters of the shader and "Blinn" basic parameters.

\section{Optimizing Model}

Optimizing model refers to reducing the complexity of the model and reducing the computational complexity of the computer. We remove the faces that are not visible due to model contact. For example, when two cubes are welded together, the transferred quadrilateral can be removed. Using the "MultrRes" modifier to adjust the vertex percentage and the number of vertices to achieve the effect of reducing the number of faces.

\section{Render to Textures}

Using "Render to Textures" can show the lighting effect. We render the light information into a map, and apply the map with the light to the scene. The render to textures decreases the time of lighting calculation and improve the simulation efficiency obviously without sacrificing rendering effect.

\section{E. Exporting the Model}

Export the constructed 3D model scene in a *.FBX format and select the default settings for importing into Unity 3D virtual engine.

\section{REALIZING INTERACTION BASED ON UNITY 3D}

Unity 3D has a good 3D rendering effect. It can achieve cross-platform development and has a rich collection of resources. Therefore, we select Unity 3D as the engine of immersion virtual training system development.

\section{F. Importing the Model}

The model file created in 3ds Max is imported into the "Assets" under the "Project" column of Unity 3D. And the model can appear as a material in the scene construction. The material in "Assets" can be directly dragged into the "Scene" view. We establish the model in the scene through this method.

G. Realizing Interaction through HTC Vive

1) Making VR Camera

Drag "CameraRig" in "Prefabs" into the scene as the camera for the VR headset. There are three parts 
in this preform corresponding to the headset and two handles. Two handles have two models respectively and are mounted "Render Model" script which is used to render the model. The "Game View" script is used to display the header, and the "Tracked Object" script is used to track the location.

Add a script to the camera and click the "Expand" button. The camera will be divided into four parts: "Origin”, “Head”, "Eye” and "Ears”. SteamVR_ControllerManager and SteamVR_TrackedObject are used to set up and detect the Vive handles. Add two sub-objects on "Origin" to represent two Vive handles. And add SteamVR_TrackedObject on them. Add SteamVR_ControllerManager to “Origin” and set handles to complete the handle integration.

2) Realizing handle control

HTC Vive handle is consisted of Application, Touchpad, System, Trigger, Grip Button. Getting the status of a key is realized through the following code.

var device=SteamVR_Controller.Input(unit);

device.GetTouchDown(SteamVR_Controller.ButtonMask)

Create a sub-object on each handle. Add SteamVR_RenderModel to the sub-object and set the "Shader" according to the requirements to synchronize the handle model in the virtual scene with the handle position in the real world.

H. Building the Project

After completing the realization of the interaction in the virtual environment, save the project as a *.unity file.

\section{Dvelopment Based on MeiTeng Platform of Virtual Maintenance Training}

MeiTeng Platform of Virtual Maintenance Training is developed by Shijiazhuang Mechanical Engineering College. It was published in 2009 officially and continues to be improved. The current version is MeiTeng 3.0. MeiTeng platform has two core functions which are training data development and training \& management. It consists of MeiTeng Data Development System and MeiTeng Virtual Maintenance Training System. MeiTeng Data Development System supports the design, development, management and publishing of virtual maintenance training data. MeiTeng Virtual Maintenance Training System can realize learning, training and assessment for construction, operation, decomposition and integration, inspection and fault diagnosis of complex equipment such as mechanical, electronic and hydraulic. MeiTeng platform can support desktop, immersion, semi-physical, touch screen and other styles of virtual maintenance training simulation. It can support a variety of interfaces as well. Therefore, this paper chooses MeiTeng platform to realize the immersive virtual maintenance training system.

\section{Establishing Training Subjects}

Select MeiTeng Equipment Virtual Maintenance Simulation System to establish the basic training subjects in virtual maintenance.

1) Establishing the project

Create a new project in MeiTeng Equipment Virtual Maintenance Simulation System and determine its name. Import the *.unity file generated by Unity 3D into the "3D scene file" column to complete the creation of the new project. Such as "Repair of a certain type of vehicle engine".

2) Construction of Operating Procedures Based on Project Engineering Tree

The new project is related to the specific operating procedures. First select the simulation content type which is divided into maintenance process simulation and fault diagnosis simulation. Secondly, select simulation mode which is divided into flow chart and lane diagram. Such as "Disassembly of engine". The simulation of the maintenance process. And select flow chart to perform the virtual maintenance process. 


\section{a) Project preparation}

Project preparation consists of scene preparation, tool preparation and device preparation. Scene preparation refers to the specific choice of virtual scenes. Tool preparation is the choice of tools could be used in a virtual maintenance process. Such as hands, screwdriver, pliers and so on. And the tools appears in the toolbox in the virtual environment. Device preparation refers to the choice of devices could be used in a virtual maintenance process. Such as jacks, generators and so on.

\section{b) Maintenance process information editing}

In the process of establishing the operation process, the basic information, prompt information and correct feedback information of each step must be established. The wrong feedback information, help information and data information can be established selectively.

Basic information editing. Firstly, determine the number, name and operator of each step. And then select interaction mode which is divided into clicking the virtual equipment and answering the question. Choose the type of interaction. Such as operation, disassembly and BIT detection. Finally determine the operation object. Such as the fifth step is "Participants flip cab". The interaction mode is clicking the cab in virtual environment through the handle. And the interaction type is operation. The operation object is the cab. (Figure 1. )

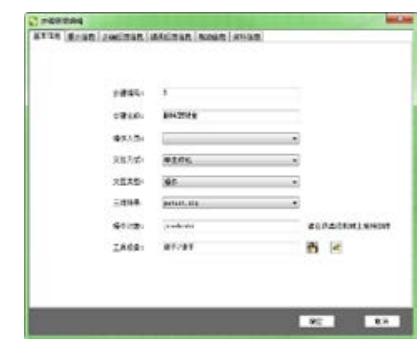

Figure 1. Basic information editing

Prompt information editing. Prompt information is divided into animation, text and file. It is used to prompt the participants to the next step in the operation. The three-dimensional scene, object name, animation name and other information should be cleared when we create animation information. For example, establish the animation prompt information for flipping cab. We drag the *.unity file into the "3D scene" column. The operation object is the cab. And the animation name is "Flipping cab animation prompt”.

Correct feedback information editing. According to the actual situation to establish feedback information. Such as "Participants flip cab correct feedback". When the participants operates correctly, the cab will be flipped over in the virtual environment.

c) Generating maintenance process

After correctly editing the information above, establish the information of every step according to the operational specification. Then generate the virtual operation process of the project. (Figure 2. )

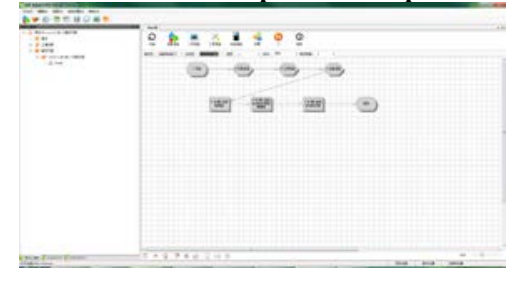

Figure 2. Generating maintenance process

3) File release and saving

When the editing to virtual maintenance operations in MeiTeng Equipment Virtual Maintenance 
Simulation System has been completed, we can choose to publish as *.proj format files. It can be directly run through the MeiTeng platform to view the animation of the training subject. We can also choose to save it in *.swn format for the realization of virtual maintenance system through MeiTeng Collaborative Equipment Virtual Maintenance Training Platform.

\section{J. Realizing Training System}

We develop the virtual maintenance training system through MeiTeng Development System for Collaborative Equipment Virtual Maintenance Training Platform.

1) Creating and connecting to the server

When the maintenance tasks need participants to operate at the same time, they can collaborate through the server. When they log in to the system, they need to fill in the name, level and other information

2) Implementation process

Create a database and connect to it.

Set up basic information. Including the basic information of equipment, such as the name. And equipment components information is mainly to build the virtual components. Personnel professional information, such as mechanical professional. Lastly, staff level information, refers to the level of participants. (Figure 3. )

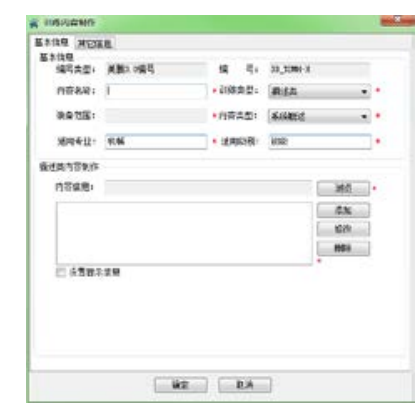

Figure 3. Set up basic information

Import training material. MEITENG development system for collaborative equipment virtual maintenance training platform has good compatibility. It can support the text, presentation, picture and other training materials. In the process of realizing immersion virtual maintenance training system, we should import the file in * .swn format generated by MeiTeng Equipment Virtual Maintenance Simulation System.

Complete training content. Select the training type, including description type, virtual maintenance operation and fault diagnosis. Select the content type, including system structure, working principle, operation and five other types.

Set up training courses. First add training packets. This process requires specific name and picture. And then the curriculum design. There are six kinds of courses, demonstration, self-learning, guided training, self-training, self-test and assessment. The training content can be dragged to the appropriate training mode. Such as building a "guided training - engine disassembly" training course.

In addition, there is training resource management module, mainly for the storage of technical information. Test management module to generate questions. Such as multiple choice questions, operation questions and others. User management module and help module. All these modules can be set according to the requirement. 


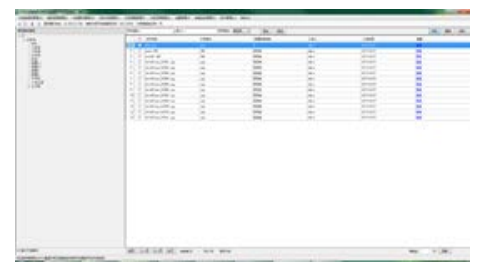

Figure 4. Training resource management module

\section{3) File release}

The system is released by the data release management module. A ResouceX format of the data packet is developed. The data package can be run in MeiTeng platform to carry out virtual maintenance training.

\section{IMPLEMENTATION AND VERIFICATION}

\section{K. Hardware and Software Requirements}

The hardware development environment of the immersive virtual maintenance system is as follows. The operating system is 64-bit Windows 7. The processor is Inter Core i5-4590 CPU@3.30GHz. The display adapter is NVIDIA GeForce GT $630 \mathrm{MB}$. The install memory (RAM) is 4.00GB. The main peripheral is HTC Vive. The software section includes: 3ds Max 2012, Unity 3D 5.1.0f3, MeiTeng Platform of Virtual Maintenance Training.

\section{Realization of Immersive Virtual Maintenance System}

This paper realizes an immersive virtual maintenance training system combined with a specific type of equipment.

Preparing before training. First, the user login. And then select the training mode, equipment and specific professional. Build and join the LAN. Choose the interactive way, which is divided into keyboard \& mouse, headset \& handle and data gloves. (Figure 5. ) All participants enter the training system. Immersive virtual maintenance training as shown in Figure 6.

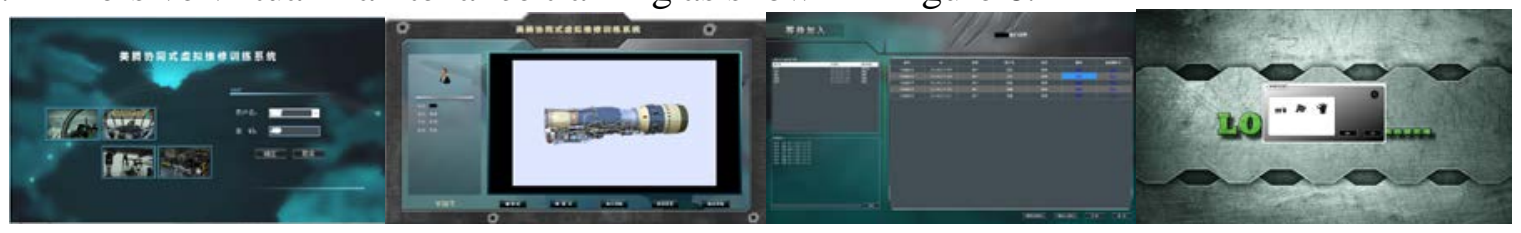

Figure 5. Preparing before training

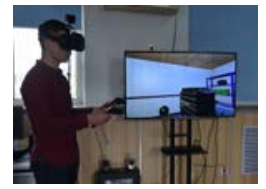

Figure 6. Immersive virtual maintenance training

The advantages of this method is obvious, such as with a short cycle, versatility and low cost. The actual operation has a good interaction, deep sense of immersion and help to improve training efficiency. It can also support desktop, touch screen, Semi-physical virtual maintenance training. MeiTeng platform can support a variety of formats of resource integration. MeiTeng offers kinds of training models. Such as self-learning, guided training, self-training, self-test and assessment. It can improve the effect of training.

The development of virtual maintenance training system based on MeiTeng platform is universal, standardlized and shareable. MeiTeng platform can achieve a variety of training models and establish a 
unified data standard. Developers can not have the knowledge of computer programming in the development process, which greatly improves the development efficiency and simplifies the development process.

\section{CoNCLUSION}

This paper presents a general method for the development of immersive virtual maintenance training system based on MeiTeng Platform of Virtual Maintenance Training. It can realize cooperative training in various training modes. This method can make up the deficiency of the traditional maintenance training methods. And it can also improve the ability of independent maintenance training and promote the development of maintenance support capacity.

\section{ACKNOWLEDGMENT}

We would like to thanks everyone who contributed to this paper. In particular, the team has developed MeiTeng Platform of Virtual Maintenance Training made this paper possible.

\section{REFERENCES}

[1] JianPing HAO, Virtual Maintenance Simulation Theory and Technology, National Defense Industry Press. Beijing, January 2008.

[2] QunXing SU, Reaserch on The Virtual Maintenance Training Platform Technology for Large Complex Equipment, Nanjing University of Science and Technology. Nanjing, December 2005.

[3] YuSong XUAN, Unity 3D Game Development, People Post Press. Beijing, June 2012.

[4] XingXin LI, A Study of Maintenance Training Model and a Platform Development, Shijiazhuang College of Mechanical Engineering, May 2010.

[5] XingXin LI, JianPing HAO, Fei YE and ZhengFan ZHAO, "The Study of Base Problems and Technique Architecture of Virtual Maintenance Training,” Microcomputer Information, vol. 26, 2010, pp. 30-32. 